\title{
PERFIL FUNCIONAL DAS SEQUELAS DE LESÃO MEDULAR NAS DIFERENTES ETIOLOGIAS
}

FUNCTIONAL PROFILE OF SPINAL INJURY SEQUELS IN DIFFERENT ETIOLOGIES

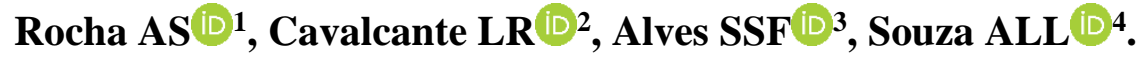

\begin{abstract}
${ }^{\mathbf{1}}$ Mestre em Saúde Coletiva pela Universidade Federal de Goiás (UFG). Docente da pós-graduação em Fisioterapia Neurofuncional no Instituto Educacional CDCS, Goiânia, Goiás, Brasil. E-mail: andrearochafisio@gmail.com. Lattes: http://lattes.cnpq.br/7145393426929408.

${ }^{2}$ Especialista em Residência em Saúde Funcional e Reabilitação pelo Centro de Reabilitação e Readaptação Henrique Santillo (CRER). Bacharel em Fisioterapia pela Universidade Estadual de Goiás (UEG), Goiânia, Goiás, Brasil. E-mail: fisio.luizarodrigues@gmail.com. Lattes: http://lattes.cnpq.br/8442486658841239.

${ }^{3}$ Mestre em Ciências da Saúde pela Universidade Federal de Goiás (UFG). Preceptora da Residência Multiprofissional em Saúde Funcional e Reabilitação no Hospital das Clínicas da Universidade Federal de Goiás, (HC-UFG), Goiânia, Goiás, Brasil. E-mail: suyasantana@gmail.com. Lattes: http://lattes.cnpq.br/5086721058061397. ${ }^{4}$ Doutora em Saúde Pública pela Universidade de São Paulo (USP). Docente no Programa de Pós-Graduação Ciências da Saúde da Faculdade de Medicina pela Universidade Federal de Goiás (UFG), Goiânia, Goiás, Brasil. E-mail: demmilima@gmail.com. Lattes: http://lattes.cnpq.br/6578713509935374.
\end{abstract}

Correspondência: Andrea Souza Rocha. Endereço: Avenida 136, nº 90, Apartamento 202, Setor Marista, Goiânia, Goiás, CEP: 74180-040. E-mail: andrearochafisio@gmail.com.

\section{Recebido: $18 / 03 / 2021$ \\ Aceito: 26/04/2021}

\section{RESUMO}

Introdução: Lesão medular (LM) é qualquer tipo de lesão neural da medula espinal podendo resultar em déficits sensoriais, motores, disfunção autonômica e esfincteriana, abaixo do nível da lesão. Objetivo: Conhecer o perfil funcional dos pacientes com LM e sua relação com diferentes etiologias. Metodologia: Estudo observacional analítico transversal, realizado no CRER. Foram aplicados questionário epidemiológico, Core Set de LM da Classificação Internacional de Funcionalidade, Incapacidade e Saúde (CIF) e Medida de Independência da Medula Espinal (SCIM III). Resultados: amostra de 65 pacientes com LM, predominando etiologia traumática $(74,2 \%)$, sexo masculino $(69,7 \%)$, idade média $37,75( \pm 15,14)$ anos, nível paraplegia $(53,1 \%)$ e lesões AIS A $(38,5 \%)$ $(p<0,05)$. As lesões não traumáticas possuem melhor prognóstico funcional. $\mathrm{O}$ controle esfincteriano e a locomoção apresentam pior funcionalidade. Conclusão: observa-se predomínio de lesões traumáticas, paraplégica, completa, em indivíduos adultos jovens masculinos, com predomínio de disfunções esfincterianas, força muscular e locomoção.

Palavras-chaves: Lesão Medular. Etiologia. Funcionalidade.

\section{ABSTRACT}

Introduction: Spinal cord injury (SCI) is any type of spinal cord neural injury that can result in sensory, motor deficits, autonomic and sphincter dysfunction, below the level of the lesion. Objective: To know the functional profile of patients with SCI and its relationship with different etiologies. Methodology: Cross-sectional observational study, carried out at CRER. Epidemiological questionnaire, Core Set of SCI of the International Classification of Functionality, Disability and Health (ICF) and Spinal Cord Independence Measure (SCIM III) were applied. Results: sample of 65 patients with SCI, traumatic etiology predominating (74.2\%), male gender $(69.7 \%)$, mean age $37.75( \pm 15.14)$ years, paraplegia level $(53.1 \%)$ and AIS A lesions (38.5\%) ( $p<0.05)$. Non-traumatic injuries have a better functional prognosis. Sphincter control and locomotion have worse functionality. Conclusion: there is a predominance of traumatic, paraplegic, complete injuries in young adult male individuals, with a predominance of sphincter dysfunctions, muscle strength and locomotion.

Keywords: Spinal Cord Injury. Etiology. Functionality. 


\section{INTRODUÇÃO}

A lesão medular (LM) caracteriza-se como uma injúria às estruturas contidas no canal medular (medula, cone medular e cauda equina), pode ser de origem traumática e não traumática. Essas lesões levam a diversos graus de déficits sensoriais, motores, disfunção autonômica e esfincteriana, abaixo do nível da lesão, o que gera uma enorme repercussão física, psíquica e social, representando um problema de saúde pública. $^{1,2,3}$

A incidência das lesões traumáticas é bastante variada em diferentes países. Entretanto, ainda não é bem definida no Brasil, pois não há um sistema de notificações eficaz dos casos e pela escassez de estudos epidemiológicos significativos. ${ }^{4,5}$ Entretanto, observa-se predomínio em indivíduos do sexo masculino, na faixa etária entre 15 e 40 anos, o que causa grande impacto socioeconômico por atingir pessoas em idade produtiva. No Brasil as principais etiologias são acidentes automobilísticos, queda de altura, acidente por mergulho em águas rasas e ferimentos por arma de fogo. ${ }^{4,5,6,7}$

Com relação às lesões não traumáticas existem poucos dados referentes às suas especificidades, porém tem-se constatado uma tendência para o aumento da incidência, decorrentes do envelhecimento da população. Os dados demográficos demonstram tratar-se em geral de uma população menos jovem, com um maior número de comorbidades, de ambos os sexos, com predomínio de paraplegia e lesões neurológicas incompletas. Sendo mais prevalentes as de etiologia degenerativa, neoplásica, vascular, autoimune e infecciosa. ${ }^{8}$

A LM, por consequência direta ou indireta de sua etiologia, leva a diferentes alterações neurológicas temporárias ou permanentes, dos tipos completa ou incompleta, resultando em sequelas motoras e sensitivas com características de tetraplegias e paraplegias. A tetraplegia apresenta comprometimento dos quatro membros e ocorre após uma lesão cervical. Enquanto a paraplegia gera uma disfunção em tronco e nos membros inferiores, resultantes de lesões torácicas, lombares ou sacrais. ${ }^{9,10,11}$

A avaliação e diagnóstico do nível e do tipo de lesão são realizados pela American Spinal Injury Association (ASIA) que desenvolveu a ASIA Imparment Scale (AIS), que permite uma caracterização do funcionamento sensitivo/motor e o tipo de lesão (completa ou incompleta) auxiliando na determinação do prognóstico e o estado atual dos pacientes. ${ }^{9,12}$

As disfunções motoras, sensoriais e autonômicas da LM criam uma série de consequências sobre o funcionamento, sejam relacionadas às funções fisiológicas, mudanças estruturais no corpo ou dificuldades em realizar atividades e na restrição da participação. ${ }^{1,13}$ De acordo com Farias e Buchalla, ${ }^{13}$ pode-se identificar o que uma pessoa realiza ou não, ao considerar as funções dos órgãos ou dos sistemas, das estruturas do corpo, das limitações de atividades e da participação social no meio ambiente onde a pessoa está inserida. 
Com isso, Riberto et al. ${ }^{14}$ pontuam que muitos aspectos das atividades e participação podem ser avaliados em uma pessoa com LM, tais como precisão, forma e tempo para realizar um movimento. Para fazer uma avaliação específica da funcionalidade em indivíduos com LM tem-se, o Core Set resumido da Classificação Internacional de Funcionalidade, Incapacidade e Saúde (CIF) para LM e a Medida da Independência da Medula Espinal versão três (SCIM III).

O Core Set resumido da CIF para LM descreve amplamente a funcionalidade de pessoas com esta condição de saúde e considera o papel dos fatores ambientais como barreiras ou facilitadores. ${ }^{15}$ Já a SCIM III é direcionada à capacidade de executar tarefas e aproxima-se de aspectos inerentes à LM. ${ }^{13}$

A LM produz grande impacto na vida da pessoa que se depara com esta nova situação. As alterações implicam na autoimagem e no reconhecimento da nova condição física. O paciente portador de LM atravessa um período de profundo aprendizado, no qual tem que lidar intensamente com suas limitações. Essas mudanças demandam readaptações físicas, emocionais e sociais, que determinam o surgimento de novos padrões de vida. ${ }^{7}$

A prevenção deste tipo de lesão exige a identificação das características dos indivíduos envolvidos, causas, níveis de lesão e deficiências, características pessoais e clínicas, além do conhecimento das situações nas quais o mesmo ocorreu, constituindo o ponto de partida para a estruturação de programas preventivos, pois a sua incidência pode variar de acordo com o local, fatores econômicos, sociais e o momento histórico. ${ }^{5,16}$

Com relação ao processo de reabilitação, percebe-se que ainda não há tratamento efetivo para restaurar as funções perdidas pela medula comprometida. Isso determina que a reabilitação e readaptação dos pacientes, por meio de uma equipe interdisciplinar, se tornaram fase obrigatória do tratamento das sequelas da LM. ${ }^{3,5}$ Ao identificar os impactos funcionais gerados pelas diferentes etiologias (traumáticas e não traumáticas) poderá ser possível traçar um prognóstico funcional e embasar adequadamente a reabilitação e a readaptação.

Contudo, percebe-se uma escassez de estudos que realizem uma correlação direta entre a funcionalidade e as etiologias das LM. Esse trabalho tem como objetivo conhecer o perfil funcional dos pacientes com sequelas de LM e sua relação com as diferentes etiologias dos indivíduos tratados em um Centro de Reabilitação.

\section{METODOLOGIA}

Trata-se de estudo observacional analítico transversal, realizado no ambulatório Centro Estadual de Reabilitação e Readaptação Dr. Henrique Santillo (CRER). Elaborado de acordo com as Diretrizes e Normas Regulamentadoras de Pesquisas Envolvendo Seres Humanos (Resolução 466/2012, do Conselho 
Nacional de Saúde), aprovado no comitê de ética do Centro de Excelência em Ensino, Pesquisa e Projetos "Leide das Neves Ferreira", sob o parecer n² 2.788.599.

A amostra foi composta por paciente com LM, tratados no CRER, que tinham mais de 3 meses de lesão, que aceitaram participar da pesquisa e que realizaram todas as avaliações propostas no estudo. Foram excluídos pacientes em fase de choque medular, menores de 18 anos, com lesões por pressão extensa, com complicações tromboembólicas e hemodinâmicas, fatores que pudessem influenciar em sua funcionalidade no momento da avaliação.

Os participantes da pesquisa foram avaliados nas dependências do CRER nos meses de agosto a outubro de 2018. Após os devidos esclarecimentos, sobre a seleção e a anuência dos mesmos, foi preenchido e assinado o Termo de Consentimento Livre e Esclarecido. Posteriormente, aplicados, na forma de entrevista e avaliação, os instrumentos de coleta: questionário epidemiológico, AIS, Core Set resumido de LM da CIF e SCIM III.

O perfil epidemiológico dos pacientes foi avaliado através de um questionário composto pelos seguintes dados: nome, prontuário, data de nascimento, idade, sexo, etiologia da lesão, data da lesão ou início dos sintomas, comorbidades associadas, escolaridade, atividades remuneradas, prática de esporte, fonte de renda, renda do familiar e estado civil.

O nível e tipo de lesão foi classificado pela AIS, escala padronizada pela ASIA, para classificação de LM. Nela é realizada uma avaliação de sensibilidade tátil e dolorosa, em 28 dermátomos e da função motora de 10 pares de miótomos bilateral. O esfíncter anal externo também é examinado com a finalidade de se determinar se a lesão é completa ou incompleta, entre os limiares de A à E, com o qual: AIS A lesão medular completa e AIS B, C e D lesões incompletas e AIS E com função motora e sensorial normal. . $^{910,11,12}$

Para avaliar de forma específica a funcionalidade de indivíduos com LM foi utilizada a SCIM III, que se trata de uma escala direcionada à capacidade de executar tarefas e aproxima-se dos aspectos inerentes à LM, podendo detectar melhorias em aspectos como respiração, habilidade para mudar a posição do corpo, andar, entre outras atividades específicas para essa população. A SCIM III é dividida em três subescalas suplementares: "Autocuidado" avalia seis tarefas e é pontuado de 0 a 20; "Respiração e controle do esfíncter" (pontuado de 0 a 40) com quatro tarefas; e "mobilidade" (pontuação de 0 a 40) com nove tarefas. $\mathrm{O}$ resultado final varia de 0 a 100 , sendo o 0 (zero) relativo à pessoa mais dependente e 100 a mais independente. ${ }^{13}$

Por fim, para categorização do perfil funcional utilizou-se o Core Set resumido da CIF para LM e a SCIM III. O Core Set da CIF para lesão medular foi publicado para classificar amplamente a funcionalidade de pessoas com LM e também considera o papel dos fatores ambientais como barreiras ou facilitadores. ${ }^{13,15}$ O Core Set resumido da CIF para LM contém 25 categorias, dessas oito derivam de Funções do Corpo, três de Estruturas do Corpo, nove de Atividades e Participação e, cinco de Fatores Ambientais. ${ }^{15}$ 
A CIF é quantificada através da mesma escala genérica: 0 (zero) não há problema; 1 (um) problema leve; 2 (dois) problema moderado; 3 (três) problema grave; 4 (quatro) problema completo; 8 (oito) não especificado e 9 (nove) não aplicável. O problema pode significar uma deficiência, limitação, restrição ou barreira, dependendo do objetivo. No caso dos fatores ambientais, este primeiro qualificador pode ser

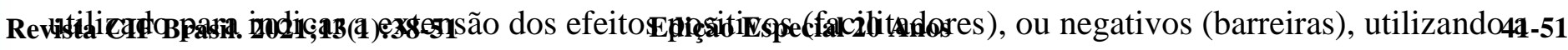
mesma escala 0-4, mas para os facilitadores utiliza-se um sinal +: por exemplo, e110+2. ${ }^{17}$

Após coletados, os dados foram organizados uma planilha eletrônica do Excelß e tratados pelo Software estatístico Statistical Package for Social Sciences (SPSS versão 23.0). Foram realizados testes de normalidade e as variáveis consideradas normais apresentadas em médias com os referidos desvios padrão e os dados não paramétricos na forma de medianas com seus respectivos intervalos de confiança.

\section{RESULTADOS}

A amostra foi composta de 65 participantes, com predomínio do sexo masculino $(69,7 \%)$, idade média 37,75 $( \pm 15,14)$ anos $(p<0,05)$. A maioria dos participantes apresentou renda média de 1 a 3 salários mínimos (54,6\%), 78,8\% pacientes não realizavam atividades remuneradas e 7,6\% são estudantes. O nível de escolaridade mais frequente foi o ensino médio completo com 27,3\%. 45,5\% dos participantes são solteiros, $71,2 \%$ não praticam esportes, $21,2 \%$ são etilistas e 4,5\% são tabagistas $(p<0,05)$. Os participantes dessa pesquisa apresentaram as seguintes comorbidades: disreflexia autonômica 18,2 \%, dislipidemia 10,6\%, pneumopatias $9,1 \%$ e depressão $7,6 \%$.

A região da lesão mais predominante foi torácica com $47 \%$ dos participantes da amostra, seguido das lesões cervicais com 45,5\% dos pacientes e as lesões lombares com 6,1\%. No nível cervical com maior amostra foi C5, já o torácico foi T12, ambos com 15,2\% da amostra cada $(\mathrm{p}<0,05)$.

Com relação às etiologias, nas lesões traumáticas foram encontradas: Acidente automobilístico $(21,2 \%)$, acidente motociclístico $(16,7 \%)$, queda $(10,6 \%)$, mergulho em água rasa $(9,1 \%)$ e perfuração por arma de fogo (16,7\%). E nas lesões não traumáticas as etiologias encontradas foram: doenças degenerativas $(9,1 \%)$, neoplásicas $(6,1 \%)$, doenças autoimunes $(1,5 \%)$ e de causa infecciosa $(7,6 \%)$. Fica demonstrado o predomínio das lesões traumáticas com 74,2\% da amostra com $p=0,020$ pelo teste de qui-quadrado.

A distribuição entre a etiologia e as regiões de LM, categorizado na TABELA 1, demostra que os acidentes automobilísticos, Mergulho em Água Rasa (MAR) apresentaram predomínio de lesões cervicais. Causas autoimune e infecciosa também apresentaram prevalência de cervicais, no entanto, sua amostra foi pequena de um e três respectivamente, enquanto nos acidentes motociclísticos e Perfuração por Arma de Fogo (PAF) ocorreu o predomínio de lesões torácicas. Por fim nas lesões de etiologia como queda, doenças degenerativas da coluna e neoplasias a incidência foi igual em cervical e torácica. 
TABELA 1 - Correlação entre etiologia e região de lesão.

Região de lesão

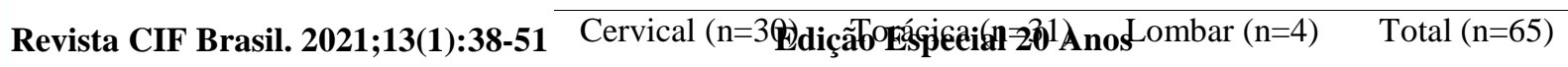

\begin{tabular}{|c|c|c|c|c|c|c|c|c|}
\hline Etiologias & $\mathrm{n}$ & $\%$ & $\mathrm{n}$ & $\%$ & $\mathrm{n}$ & $\%$ & $\mathrm{n}$ & $\%$ \\
\hline Acidente Automobilístico & 8 & 57,1 & 4 & 28,6 & 2 & 14,3 & 14 & 21,2 \\
\hline Acidente Motociclístico & 2 & 18,2 & 8 & 72,7 & 1 & 9,1 & 11 & 16,7 \\
\hline Queda & 3 & 42,9 & 3 & 42,9 & 1 & 14,3 & 7 & 10,6 \\
\hline MAR & 6 & 100 & 0 & 0 & 0 & 0 & 6 & 9,1 \\
\hline PAF & 2 & 18,2 & 9 & 81,8 & 0 & 0 & 11 & 16,7 \\
\hline Degenerativas & 3 & 50 & 3 & 50 & 0 & 0 & 6 & 9,1 \\
\hline Neoplasias & 2 & 50 & 2 & 50 & 0 & 0 & 4 & 6,1 \\
\hline Autoimunes & 1 & 100 & 0 & 0 & 0 & 0 & 1 & 1,5 \\
\hline Infecciosas & 3 & 60 & 2 & 40 & 0 & 0 & 5 & 7,6 \\
\hline
\end{tabular}

\section{Legenda: * Teste Qui-quadrado de Pearson.}

Com relação à classificação das lesões em completas e incompletas, a AIS A foi predominante com ocorrência de 38,5\%, seguido de AIS C com 27,7\%, AIS D com 20\%, AIS B com 9,2\% e, por fim, a menos prevalente AIS E com 4,6\%. A SCIM III apresentou um score médio de 63,49( $\pm 23,74)$. Na correlação desses dois dados, a SCIM demonstrou-se com melhor score funcional em AIS D 84,31( $\pm 11,19)$, seguido de AIS E 79,33( $\pm 35,8)$, AIS C 73,17( $\pm 13,14)$, AIS A 48,32( $\pm 21,7)$ e AIS B 44,67( $\pm 19,08)$ com $p=0,000$ pelo teste de Tau_b de Kendall.

Outra correlação realizada foi entre as regiões de lesão, a AIS e a SCIM III. Pode-se perceber que a melhor funcionalidade e atribuída para lesões lombares com média de 80,25( $\pm 10,69)$, que apresentaram

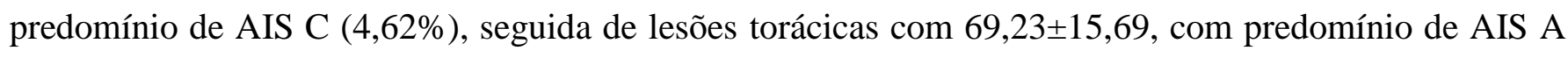
$(23,08 \%)$ e lesões cervicais com 55,33( $\pm 28,89)$ com distribuição igual em AIS A e D $(15,38 \%)$. 
Ao realizar as correlações entre Etiologia e AIS percebeu-se significância estatística com $p=0,013$, demonstrando que nas lesões provocadas por acidente automobilístico, motociclísticos e PAF ocorrem com predomínio de AIS A. Já em lesões causadas por queda, doenças degenerativas, infecciosas e doenças autoimunes há a prevalência de AIS C. Nas LM provocadas por MAR ocorreu mesma distribuição em AIS B e D. As lesões de causas neoplásicas obtiveram predomínio de AIS D, como demonstrado na TABELA 2.

TABELA 2 - Correlação entre AIS e etiologias.

\begin{tabular}{|c|c|c|c|c|c|c|}
\hline & \multicolumn{5}{|c|}{ AIS } & \multirow{3}{*}{$p$} \\
\hline & A & B & $\mathrm{C}$ & $\mathrm{D}$ & $\mathrm{E}$ & \\
\hline Etiologias & $\%(\mathrm{n})$ & $\%(\mathrm{n})$ & $\%(\mathrm{n})$ & $\%(\mathrm{n})$ & $\%(\mathrm{n})$ & \\
\hline Acidente Automobilístico & $35,7(5)$ & $7,1(1)$ & $21,4(3)$ & $28,6(4)$ & $7,1(1)$ & \multirow{9}{*}{$0,013 *$} \\
\hline Acidente Motociclístico & $72,7(8)$ & $9,1(1)$ & $9,1(1)$ & $9,1(1)$ & $0(0)$ & \\
\hline Queda & $14,3(1)$ & $0(0)$ & $71,4(5)$ & $0(0)$ & $14,3(1)$ & \\
\hline MAR & $16,7(1)$ & $33,3(2)$ & $16,7(1)$ & $33,3(2)$ & $0(0)$ & \\
\hline PAF & $81,8(9)$ & $0(0)$ & $18,2(2)$ & $0(0)$ & $0(0)$ & \\
\hline Degenerativas & $16,7(1)$ & $16,7(1)$ & $33,3(2)$ & $16,7(1)$ & $16,7(1)$ & \\
\hline Neoplasias & $0(0)$ & $25(1)$ & $0(0)$ & $75(3)$ & $0(0)$ & \\
\hline Autoimunes & $0(0)$ & $0(0)$ & $100(1)$ & $0(0)$ & $0(0)$ & \\
\hline Infecciosas & $0(0)$ & $0(0)$ & $60(3)$ & 40(2) & $0(0)$ & \\
\hline
\end{tabular}

Legenda: * Teste Qui-quadrado de Pearson.

A análise da correlação dos domínios da SCIM III com a etiologia pode ser observada na TABELA 3. Nas subescalas "autocuidado" e "sistema respiratório e esfíncteres" verifica-se que a etiologia queda

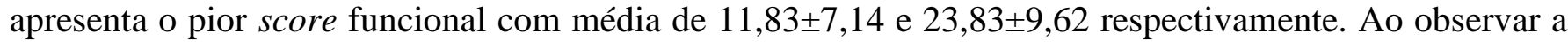
"mobilidade" desses pacientes verifica-se que em todas as etiologias essa função está prejudicada, com o pior score funcional atribuído pra PAF com média de 14,27 $\pm 8,45(p<0,05)$. Por fim, o score total da SCIM III foi pior em MAR $(49,83 \pm 24,86)$, contudo, o score total não apresentou uma relação estatisticamente significativa com as etiologias $(p>0,05)$.

O perfil funcional da amostra, baseado no Core Set resumido da CIF encontra-se relatado nas FIGURAS 1 e 2. 
TABELA 3 - Correlação entre SCIM III e etiologia.

SCIM III

Rer

\begin{tabular}{lclc}
\hline Autocuidado & $\begin{array}{c}\text { Sistema respiratório } \\
\text { esfíncteres }\end{array}$ & Mobilidade & Total
\end{tabular}

\begin{tabular}{|c|c|c|c|c|c|c|c|c|c|}
\hline \multirow[t]{2}{*}{ Etiologia } & $\%$ & Média & & Média & & Média & & Média & \\
\hline & $(\mathrm{n})$ & $( \pm \mathrm{DP})$ & $p$ & $( \pm \mathrm{DP})$ & $p$ & $( \pm \mathrm{DP})$ & $p$ & $( \pm \mathrm{DP})$ & $p$ \\
\hline \multirow[t]{2}{*}{ Acidente Automobilístico } & 21,5 & 13,36 & & 27,29 & & 19,43 & & 60,07 & \\
\hline & (14) & $( \pm 7,92)$ & & $( \pm 9,55)$ & & $( \pm 15,18)$ & & $( \pm 31,29)$ & \\
\hline \multirow[t]{2}{*}{ Acidente Motociclístico } & 16,9 & 17,18 & & 32,27 & & 19,18 & & 69 & \\
\hline & (11) & $( \pm 4,94)$ & & $( \pm 5,14)$ & & $( \pm 7,91)$ & & $( \pm 15,18)$ & \\
\hline \multirow[t]{2}{*}{ Queda } & 10,8 & 18,14 & & 32,43 & & 25,71 & & 76,29 & \\
\hline & (7) & $( \pm 2,8)$ & & $( \pm 8,72)$ & & $( \pm 9,41)$ & & $( \pm 17,19)$ & \\
\hline \multirow[t]{2}{*}{ MAR } & 9,2 & 11,83 & & 23,83 & & 20,83 & & 49,83 & \\
\hline & (6) & $( \pm 7,14)$ & & $( \pm 9,62)$ & & $( \pm 22,6)$ & & $( \pm 24,86)$ & \\
\hline \multirow[t]{2}{*}{$\mathrm{PAF}$} & 16,9 & 13,36 & $0,000 *$ & 29,36 & $0,005^{*}$ & 14,27 & $0,032 *$ & 57 & $0,387 * *$ \\
\hline & $(11)$ & $( \pm 7,13)$ & & $( \pm 8,31)$ & & $( \pm 8,45)$ & & $( \pm 22,78)$ & \\
\hline \multirow[t]{2}{*}{ Degenerativas } & 9,2 & 13,5 & & 30,67 & & 20,50 & & 54,67 & \\
\hline & (6) & $( \pm 8,43)$ & & $( \pm 9,79)$ & & $( \pm 15,98)$ & & $( \pm 29,23)$ & \\
\hline \multirow[t]{2}{*}{ Neoplasias } & 6,2 & 18,25 & & 33,5 & & 24,75 & & 76,5 & \\
\hline & (4) & $( \pm 1,5)$ & & $( \pm 10,38)$ & & $( \pm 11,5)$ & & $( \pm 21,06)$ & \\
\hline \multirow[t]{2}{*}{ Autoimunes } & 1,5 & 12 & & 37 & & 19 & & 68 & \\
\hline & $(1)$ & & & & & & & & \\
\hline \multirow[t]{2}{*}{ Infecciosas } & 7,7 & 16,8 & & 33,6 & & 22,6 & & 73 & \\
\hline & (5) & $( \pm 3,96)$ & & $( \pm 5,6)$ & & $( \pm 8,79)$ & & $( \pm 11,68)$ & \\
\hline \multirow[t]{2}{*}{ Total } & 100 & 14,94 & $0,000 *$ & 30,05 & $0,005^{*}$ & 19,98 & $0,032 *$ & 63,49 & $0,387 * *$ \\
\hline & $(65)$ & $( \pm 6,4)$ & & $( \pm 8,46)$ & & $( \pm 12,58)$ & & $( \pm 23,74)$ & \\
\hline
\end{tabular}

Legenda: *Teste Qui-quadrado **Teste Anova. 


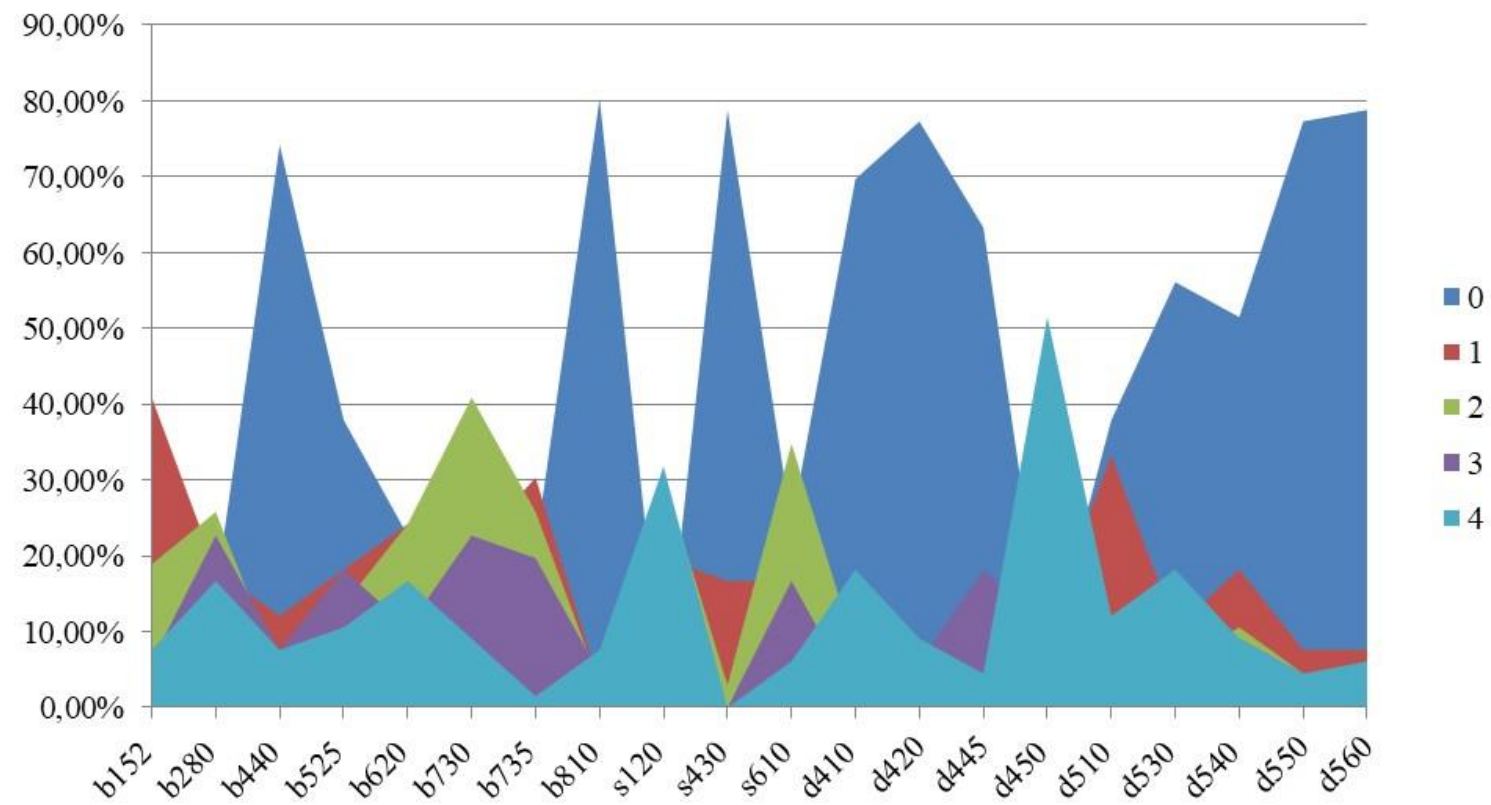

FIGURA 1 - Core Set resumido da CIF para LM: Funções do Corpo, Estruturas do Corpo e Atividades e Participação.

$* p<0,05$ no Teste Qui-quadrado

b152 - Funções emocionais; b280- Sensação de dor; b440- Funções da respiração; b525- Funções de defecação; b620- Funções urinárias; b730- Funções relacionadas à força muscular; b735- Funções relacionadas ao tônus muscular; b810- Funções protetoras da pele; s120- Medula espinhal e estruturas relacionadas; s430- Estrutura do aparelho respiratório; s610- Estrutura do aparelho urinário; d410- Mudar de posição básica do corpo; d420Transferir a própria posição; d445-Uso da mão e do braço; d450-Andar; d510- Lavar-se; d530- Cuidados relacionados aos processos de excreção; d540- Vestir-se; d550- Comer; d560- Beber.

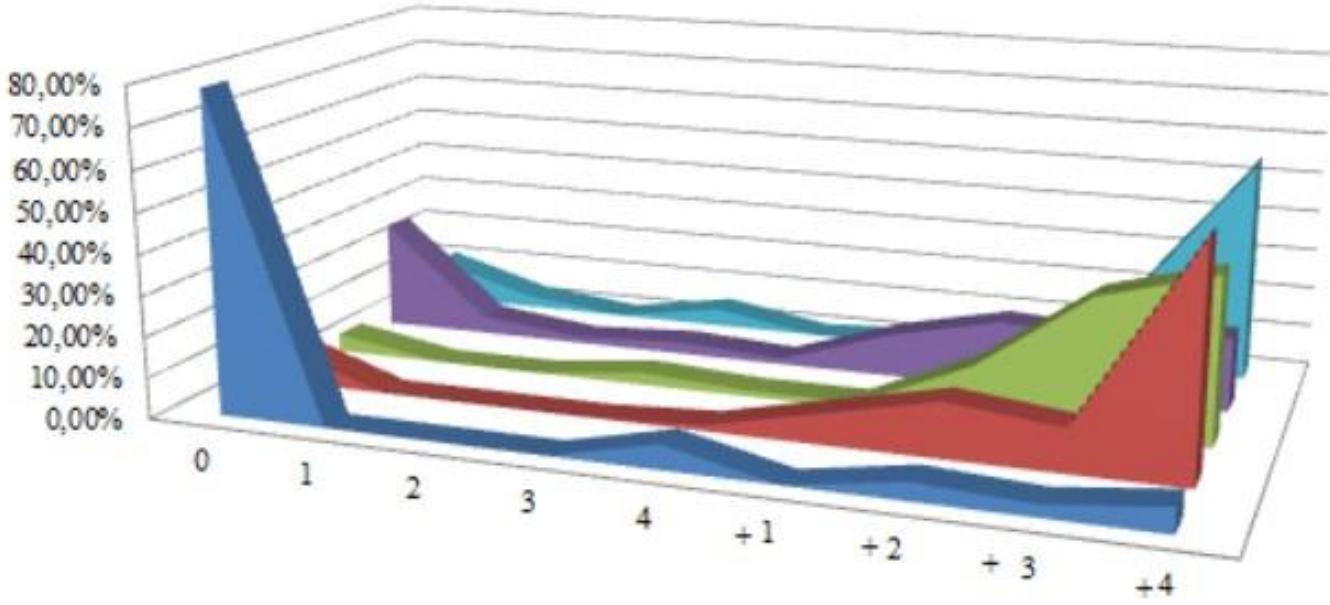

\begin{tabular}{|c|ccccccccc|}
\cline { 2 - 10 } \multicolumn{1}{c|}{} & 0 & 1 & 2 & 3 & 4 & +1 & +2 & +3 & +4 \\
\hline$=e 115$ & $78,80 \%$ & $0 \%$ & $0 \%$ & $0 \%$ & $6,10 \%$ & $0 \%$ & $4,50 \%$ & $3 \%$ & $6,10 \%$ \\
$=e 120$ & $9,10 \%$ & $0 \%$ & $0 \%$ & $0 \%$ & $1,50 \%$ & $7,60 \%$ & $13,60 \%$ & $10,60 \%$ & $56,10 \%$ \\
$=e 310$ & $4,50 \%$ & $0 \%$ & $0 \%$ & $3 \%$ & $1,50 \%$ & $1,50 \%$ & $13,60 \%$ & $33,30 \%$ & $40,90 \%$ \\
$=\mathrm{N}=340$ & $27,30 \%$ & $4,50 \%$ & $1,50 \%$ & $3 \%$ & $1,50 \%$ & $10,60 \%$ & $16,70 \%$ & $15,20 \%$ & $18,20 \%$ \\
$=\mathrm{N}=355$ & $10,60 \%$ & $3 \%$ & $0 \%$ & $4,50 \%$ & $0 \%$ & $1,50 \%$ & $4,50 \%$ & $19,70 \%$ & $54,50 \%$ \\
\hline
\end{tabular}

FIGURA 2 - Core Set resumido da CIF para LM: Fatores Ambientais.

$* p=0,000$ no Teste Qui-quadrado

e115 - Produtos e tecnologias para uso pessoal na vida diária; e120 - Produtos e tecnologias para mobilidade e transporte pessoal em ambientes interno e externo; e310 - Família próxima; e340 Cuidadores e assistentes pessoais; e355 - Profissionais de saúde. 
Quanto às "funções de respiração" (b440), às "estruturas do aparelho respiratório" (s430), às "funções protetoras da pele" (b810) e às "funções de defecação" (b525) dos pacientes avaliados, não apresentaram alterações funcionais, com predomínio do descritor 0 em, 74,2\%, 78,8\%, 80,3\% e 37,9\% respectivamente. As funções do corpo "b152" (Funções emocionais) e "b735" (Funções relacionadas ao tônus muscular) apresentaram um problema leve para 40,9\% e 30,3\% dos casos respectivamente $(\mathrm{p}<0,05)$.

Já as "estruturas do aparelho urinário" (s610) 34,8\% e "estrutura da medula espinhal e estruturas relacionadas" (s120) 31,8\% apresentaram disfunção moderado e completa respectivamente. Com 40,9\% as “funções relacionadas à força muscular" (b730) apresentam um problema moderado. Enquanto, as "funções sensações de dor" (b280) e "Funções urinárias" (b620) ficaram bem distribuídas em todos os descritores, e não obtiveram correlação estatisticamente significativa $(p>0,05)$.

As atividades e participação que determinam a independência desses indivíduos tais como: "mudar de posição básica do corpo" (d410) com 69,7\%, "transferir a própria posição" (d420) com 77,3\%, "uso da mão e do braço" (d445) com 63,3\%, "lavar-se" (d510) com 37,9\%; "cuidados relacionados aos processos de excreção" (d530) com 56,1\%; "vestir-se" (d540) com 51,5\%; “comer" (d550) com 77,3\% e "beber" (d560) com 78,8\%, não apresentaram disfunção. Entretanto, o "andar" (d450) demonstrou uma disfunção completa com $51,5 \%(\mathrm{p}<0,05)$.

$\mathrm{Na}$ descrição dos fatores ambientais, pode se perceber que a maioria da amostra não necessitava de "produtos e tecnologias para uso pessoal na vida diária" (e115) 78,8\%, em contraponto a maioria necessitava de "produtos e tecnologias para mobilidade e transporte pessoal em ambientes interno e externo" (e120) 56,1\%. Em relação à "família próxima” (e310) 40,9\% caracterizaram com facilitador total, e 27,3\% não possuíam "cuidadores e assistentes pessoais" (e340). Os "profissionais de saúde" (e355) também foram caracterizados em sua maioria com facilitadores com 54,5\%.

\section{DISCUSSÃO}

Os dados obtidos nesse estudo demonstram que os 65 pacientes possuem um perfil epidemiológico caracterizado por predomínio de homens jovens. Pode-se observar também, prevalência de lesões traumáticas completas, o que demonstra um maior comprometimento funcional para essa população, com maiores restrições funcionais no que diz respeito às estruturas esfincterianas, à força muscular e à locomoção.

Corroborando com aos dados descritos na literatura, o perfil desses pacientes atestou o predomínio de LM em indivíduos do sexo masculino, com idade produtiva, com lesões de origem traumática, ao nível paraplégico e com lesões completas. . $2,5,4,11,19$

Com relação ao nível de lesão, de forma específica, o presente estudo verificou predomínio de lesões 
torácicas, corroborando com os dados de Custodio et al..$^{5}$ que relatou, para as LM traumáticas, maior incidência nas regiões cervicais e de transição toracolombar, com tudo predominando as lesões torácicas. ${ }^{5,19}$ Enquanto nas lesões não traumáticas, Almeida et al. ${ }^{8}$ descrevem uma relação de paraplegia mais evidente do que a tetraplegia, mas no presente estudo não foi evidenciado essa prevalência. Observa-se maior incidência de cervicais, porém a representação da amostra de lesões não traumáticas desse estudo é pequena para fazer essa determinação.

Para a discriminação do tipo de lesão, completa ou incompleta, verifica-se prevalência de lesões completas, ou seja, AIS A. ${ }^{18,19,20}$ Principalmente em etiologias traumáticas provocadas por acidente automobilístico, motociclístico e PAF. ${ }^{1,10,18}$ Isso pode ser justificado pela fisiopatologia do trauma, que ocorre em dois momentos: lesão primaria e lesão secundária. A lesão primaria é do tipo compressivo contusivo, que leva ao deslocamento, fratura e mutilação dos elementos da coluna vertebral. E a lesão secundaria é ocasionada por fatores vasculares, celulares e moleculares depois da lesão. ${ }^{21}$ Em contrapartida, ao se descrever as lesões não traumáticas percebe-se predomínio de lesões incompletas AIS C e AIS D, ${ }^{8}$ nas principais etiologias (degenerativas, neoplásicas e infecciosas) ${ }^{1,8,18}$ por apresentarem um evento estressor inespecífico e único.

Percebe-se de forma geral que em presença de lesões completas e com níveis cervicais os scores funcionais da SICM III são piores, quando comparados às lesões incompletas e com níveis torácicos e lombares. Com isso, pode-se descrever um prognóstico funcional para estes pacientes através da utilização da AIS, que determina tipo e nível de lesão.

Quando analisada a etiologia com os domínios da SCIM III, pode-se perceber que as etiologias neoplásicas e infecciosas apresentaram melhor pontuação em "autocuidado" e "sistema respiratório e esfíncteres" respectivamente. No que diz respeito à "mobilidade" toda a amostra se apresentou com restrições nessa atividade, com pior funcionalidade atribuída a PAF. No entanto, o score total da escala não apresentou uma correlação estatisticamente significativa com as etiologias. Com possível justificativa pela falta de representatividade da amostra.

Sendo assim, infere-se que nas lesões traumáticas, que obtiveram mais lesões completas, os indivíduos possuem uma maior incapacidade funcional, trazendo restrições à participação desses indivíduos. Entretanto o contrário é valido para as lesões não traumáticas, pois possuem mais lesões incompletas, ou seja, scores funcionais melhores. ${ }^{18,22}$

O perfil funcional e as principais restrições de atividade e participação dos pacientes com LM, foram classificadas pelo Core Set resumido da CIF. Para Vall et al. ${ }^{23}$ essa aplicação da CIF em indivíduos com lesão medular demonstrou uma série de incapacidades e limitações relacionadas às funções corporais, estruturas do corpo atividades e participação e fatores ambientais. Tais dados discordam dos dados encontrados nesta pesquisa que demonstraram que as maiorias das categorias do Core Set da CIF não 
apresentaram disfunções importantes, exceto em "funções emocionais" (b152); "funções relacionadas ao tônus muscular" (b735); "funções relacionadas à força muscular" (b730); "estrutura do aparelho urinário" (s610); “estrutura da medula espinhal e estruturas relacionadas" (s120) e "andar" (d450).

No que diz respeito às "funções emocionais" os indivíduos desta pesquisa apresentaram, em sua maioria, um problema leve, levando em consideração que 7,6\% dessa amostra são de depressivos. Ao considerar esse fato, Borges et al. ${ }^{24}$ relataram que a LM é caracterizada pela perda da integridade física e mudanças da imagem corporal, o que pode levar à desestruturação psíquica.

As "funções relacionadas ao tônus muscular" se mostraram com uma leve disfunção, ou seja, a intensidade da espasticidade, assim como a frequência dos automatismos, nesta população, não gerou incapacidade ou dificuldades na realização das atividades de vida diária como as transferências, troca do vestuário e o posicionamento. ${ }^{1}$

No entanto, a "função relacionada à força muscular" demonstrou um problema moderado. Nunciato

et al. ${ }^{25}$ descreve que a funcionalidade está associada diretamente com o pico de torque muscular, ou seja, quanto melhor a força muscular melhor o desempenho e independência desses indivíduos.

Com relação, às "estruturas do aparelho urinário" e "estruturas da medula espinhal" este estudo apresentou disfunções moderadas e completas respectivamente, justificado pela maior prevalência de AIS A, que se caracteriza pela ausência dos controles esfincterianos e secção completa da medula. ${ }^{9,10,11}$

Na atividade e participação "andar” ocorreu um predomínio de disfunções completas, caracterizado pela amostra que possuía um percentual maior de indivíduos com LM do tipo paraplegia completa. Esse fato também confirmou o achado da SCIM III que demonstra um score baixo no domínio de "mobilidade". Dessa forma a maioria da amostra necessitava dos fatores ambientais "produtos e tecnologias para mobilidade e transporte pessoal em ambientes interno e externo", caracterizados como facilitadores completos.

As relações sócias podem possuir um caráter facilitador completo ou barreira. "Família próxima" e "profissionais da saúde", são descritos neste estudo como facilitadores completos, o que pode denotar a dependência direta dos indivíduos do ciclo familiar e de cuidados comprometendo sua independência. Contudo sabe-se, que uma boa rede do apoio pode ajudar o paciente a estabelecer estratégias de enfrentamento, especialmente o apoio familiar e o companheirismo das amizades. ${ }^{9}$

Deste modo acredita-se que dentro do diagnóstico funcional a LM apresenta maiores restrições de atividade e participação no que diz respeito à estrutura esfincteriana, a força muscular e a locomoção.

\section{CONSIDERAÇÕES FINAIS}

O conhecimento da epidemiologia de LM é importante não só para o planejamento de recursos, mas também para o tratamento, a reabilitação e a readaptação dos pacientes. Embora este estudo não 
compreenda uma representação da epidemiologia e todas as lesões, por estar restrito a uma única instituição, observa-se uma consonância com a literatura no que diz respeito ao predomínio de lesões traumáticas, do tipo paraplégico, de secção completa, em indivíduos adultos jovens do sexo masculino, com predomínio de disfunções esfincterianas, de força muscular e de locomoção, sendo necessária a utilização de dispositivos de locomoção.

Pode se observar também que as lesões não traumáticas possuem um prognóstico funcional melhor em comparação com as lesões traumáticas. Contudo fazem-se necessários mais estudos para confirmar este achado.

Por fim, sugere-se que as condutas e protocolos terapêuticos, de reabilitação e readaptação, levem em consideração a correlação entre etiologia e funcionalidade, no sentido de nortear suas práticas e tornálas mais eficientes e direcionadas para o incremento da funcionalidade ajustada ao potencial de cada paciente.

\section{REFERÊNCIAS}

1. Ministério da Saúde. Diretrizes de Atenção à Pessoa com Lesão Medular. Secretaria de Atenção à Saúde. Departamento de Ações Programáticas Estratégicas. - Brasília: Ministério da Saúde, 2013. Disponível em:

http://bvsms.saude.gov.br/bvs/publicacoes/diretrizes_atencao_pessoa_lesao_medular.pdf.

2. Santos TSC, Guimarães RM, Boeira SF. Epidemiologia do trauma raquimedular em emergências públicas no município do Rio de Janeiro. Esc Anna Nery. 2012;16(4):747-753. DOI: https://doi.org/10.1590/S1414-81452012000400015.

3. Gross-Hemmi MH, Post MWM, Ehrmann C, Fekete C, Hasnan N, Middleton JW, et al. Study Protocol of the International Spinal Cord Injury (InSCI) Community Survey. Am J Phys Med Rehabil. 2017;96(2 Suppl 1):S23-S34. DOI: https://doi.org/10.1097/PHM.0000000000000647.

4. Passos ROP, Pereira CU, Santos ACL, Santos JÁ, Sousa AH. Epidemiologia do Traumatismo Raquimedular em Idosos. J Bras Neurocirurg. 2013; 24(4):300-35. DOI: https://doi.org/10.22290/jbnc.v24i4.1447.

5. Custódio NRO, Carneiro MR, Feres CC, Lima GHS, Jubé MRR, Watanabe LE, et al. Lesão medular no Centro de Reabilitação e Readaptação Dr. Henrique Santillo (CRER- GO). Coluna/Columna. 2009;8(3):265-268. DOI: https://doi.org/10.1590/S1808-18512009000300005.

6. Costa RC, Caliri MHL, Costa LS, Gamba MA. Fatores associados à ocorrência de úlcera por pressão em lesados medulares. Rev Neurocienc. 2013;21(1):60-68. DOI: https://doi.org/10.34024/rnc.2013.v21.8206.

7. Schoeller SD, Bitencourt RN, Leopardi MT, Pires DP, Zanini MTB. Mudanças na vida das pessoas com lesão medular adquirida. Rev. eletrônica enferm. 2012;14(1):95-103.

8. Almeida C, Ferreira A, Faria F. Lesões medulares não traumáticas: Caracterização da população de um Centro de Reabilitação. Revista da Sociedade Portuguesa de Medicina Física e de Reabilitação. 2011;20(1):34-37. DOI: https://dx.doi.org/10.25759/spmfr.5.

9. American Spinal Injury Association. International standarts for neurological classification of spinal cord injury. Atlanta (US): American Spinal Injury Association; 2011. Disponível em: http://www.asia-spinalinjury.org/publications/59544_sc_Exam_Sheet_r4.pdf. 
10. França ISX, Coura AS, França EG, Basílio NNV, Souto RQ. Qualidade de vida de adultos com lesão medular: um estudo com WHOQOL-bref. Rev. esc. enferm. USP. 2011;45(6):1364-1371. DOI: https://doi.org/10.1590/S0080-62342011000600013.

11. Freitas DMO. Trauma raquimedular: epidemiologia e implicações decorrentes desta patologia. Caderno Saúde e Desenvolvimento. 2016;9(5):45-59.

12. Neves MAO, Mello AO, Antonioli MP, Souza R, Freitas MRG. Escalas clínicas e funcionais no gerenciamento de indivíduos com Lesões Traumáticas da Medula Espinhal. Rev Neurocienc. 2007; 15(3):234-239. DOI: https://doi.org/10.34024/rnc.2007.v15.8693.

13. Farias N, Buchalla CM. A Classificação Internacional de Funcionalidade, Incapacidade e Saúde da Organização Mundial da Saúde: Conceitos, Usos e Perspectivas. Rev Bras Epidemiol. 2005;8(2):187193. DOI: https://dx.doi.org/10.1590/S1415-790X2005000200011.

14. Riberto M, Tavares DA, Rimoli JRJ, Castineira CP, Dias RV, Franzoi AC, et al. Validation of the Brazilian version of the Spinal Cord Independence Measure III. Arq Neuropsiquiatr. 2014;72(6):439444. DOI: https://doi.org/10.1590/0004-282X20140066.

15. Viana PC. Validação do instrumento baseado no core set resumido da Classificação Internacional de Funcionalidade, Incapacidade e Saude (CIF) para indivíduos com lesão medular aguda traumática. Dissertação de mestrado da Universidade de São Paulo, 2015. DOI: https://dx.doi.org/10.11606/D.22.2016.tde-08032016-151059.

16. Joseph C, Delcarme A, Vlok I, Wahman K, Phillips J, Nilsson Wikmar L. Incidence and aetiology of traumatic spinal cord injury in Cape Town, South Africa: a prospective, population-based study. Spinal Cord. 2015;53(9):692-696. DOI: https://dx.doi.org/10.1038/sc.2015.51.

17. Organização Mundial da Saúde. CIF: Classificação Internacional de Funcionalidade, Incapacidade e Saúde. Trad. do Centro Colaborador da Organização Mundial da Saúde para a Família de Classificações Internacionais. São Paulo: EDUSP; 2003.

18. Equebal A, Anwer S, Kumar R. The prevalence and impact of age and gender on rehabilitation outcomes in spinal cord injury in India: a retrospective pilot study. Spinal Cord. 2013;51:409-412. DOI: https://dx.doi.org/10.1038/sc.2013.5.

19. Cieza A, Kirchberger I, Biering-Sørensen F, Baumberger M, Charlifue S, Post MW, et al. ICF core sets for individuals with spinal cord injury in the long-term context. Spinal Cord. 2010;48(4):305312. DOI: https://dx.doi.org/10.1038/sc.2009.183.

20. Karamouzian S, Saeed A, Ashraf-Ganjouei K, Ebrahiminejad A, Dehghani MR, Asadi AR. The neurological outcome of spinal cord injured victims of the Bam earthquake, Kerman, Iran. Archives of Iranian medicine. 2010;13(4):351-4. PMID: 20597570.

21. Nunes DM, Morais CR, Ferreira CG. Fisiopatologia da lesão medular: uma revisão sobre os aspectos evolutivos da doença. Getec. 2017;6(13):87-103

22. Scivoletto G, Bonavita J, Torre M, Baroncini I, Tiberti S, Maietti E, et al. Observational study of the effectiveness of spinal cord injury rehabilitation using the Spinal Cord Injury-Ability Realization Measurement Index. Spinal cord. 2016;54(6):467-72. DOI: https://dx.doi.org/10.1038/sc.2015.153.

23. Vall J, Costa CMDC, Pereira LF, Friesen TT. Application of International Classification of Functioning, Disability and Health (ICF) in individuals with spinal cord injury. Arquivos de neuropsiquiatria. 2011;69(3):513-518. DOI: https://doi.org/10.1590/S0004-282X2011000400020.

24. Borjes AMF, Brignol P, Schoeller SD, Bonetti A. Percepção das pessoas com lesão medular sobre a sua condição. Rev Gaúcha Enferm. 2012;33(3):119-125. DOI: https://doi.org/10.1590/S198314472012000300016.

25. Nunciato AC, Pastrelo D, Leite RD, Prestes J, Medalha CC. Treinamento de força e treinamento funcional em adolescente lesado medular - Relato de caso. ConScientiae Saúde. 2009;8(2):281-288. DOI: https://doi.org/10.5585/conssaude.v8i2.1644. 